\title{
MiR-761 Promotes Progression and Metastasis of Non-Small Cell Lung Cancer by Targeting ING4 and TIMP2
}

\author{
An Yan ${ }^{\mathrm{a}}$ Chunyu Yang ${ }^{\mathrm{a}}$ Zhibo Chen ${ }^{\mathrm{b}}$ Chunhong Li ${ }^{\mathrm{a}} \quad \mathrm{Li}_{\mathrm{Cai}}{ }^{\mathrm{a}}$ \\ aDepartment of Internal Medical Oncology, bepartment of Pharmacy Intravenous Admixture Service, \\ Harbin Medical University Cancer Hospital, Harbin, Heilongjiang Province, China
}

\section{Key Words}

miR-761 • NSCLC • Metastasis • Proliferation • ING4 • TIMP2

\begin{abstract}
Background/Aims: The aim of this study was to investigate the role of microRNA miR-761 in the progression and metastasis of non-small cell lung cancer (NSCLC), and the mechanisms by which miR-761 regulates cell proliferation and metastatic activity of NSCLC cell lines. Methods: Quantitative real-time PCR (qRT-PCR) was used to assess miR-761 expression in NSCLC serum and tissue. MTT, wound healing, and transwell assays were performed to examine the role of miR-761 in regulation of cell proliferation and metastatic activity in NSCLC cell lines. In addition, the correlations of miR-761 expression with clinical-pathologic factors were statistically analyzed. Finally, we investigated whether miR-761 promotes proliferation and metastasis in NSCLC cell lines by targeting ING4 (inhibitor of growth family, member 4) and TIMP2 (tissue inhibitor of metalloproteinase 2). Results: MiR-761 was significantly upregulated in both NSCLC serum and tissues as compared to normal participants and paired noncancerous tissues respectively. Ectopic expression of miR-761 promoted cell proliferation and metastasis in $\mathrm{H} 460$ cells, while miR-761 inhibitor reduced proliferation rates and metastasis in $\mathrm{H} 23$ cells. Furthermore, luciferase reporter assay and functional analyses indicated that miR-761 directly targeted ING4 and TIMP2. Conclusion: miR-761 promotes progression and metastasis of NSCLC by targeting ING4 and TIMP2.
\end{abstract}

\section{Introduction}

Non-small cell lung cancer (NSCLC) accounts for approximately 85\% of all cases of lung cancer, the leading cause of cancer-related mortality worldwide [1,2]. Lung cancer A. Yan and C. Yang contributed equally to this work. 
may spread to both intrathoracic and extrathoracic areas in late stages of disease [3]. Intrathoracic spread of lung cancer is caused by either direct extension or lymphatic spread, and lung cancer metastasis may travel through airways to distant tissues, such as the bones, liver, adrenal glands, and brain and spinal cord [4]. At initial diagnosis, $25 \%$ of lung cancer patients have regional metastasis, and 55\% of patients have distant spread of disease [5]. Smoking, including passive smoking, has long been considered as the main cause of lung cancer in both genders [6]. Interestingly, a recent study found an increase in the proportion of NSCLC patients who never smoked, revealing the role of specific molecular and genetic tumor characteristics in initiation and progression of NSCLC [3, 7].

microRNAs (miRNAs) are a class of small non-coding RNAs 18-25 nucleotides long that negatively regulate gene expression at the posttranscriptional level [8]. As previously suggested, disrupted microRNA expression in lung cancer may be involved in regulation of cell proliferation, apoptosis, and angiogenesis [9-11]. miRNAs may also serve as lung cancer biomarkers because of their unique expression profiles, remarkable stability, and presence in body fluids [12]. In addition, miRNA profiling of lung cancer has been used to examine the correlation of miRNA expression with clinical-pathological parameters, and to predict patient survival [13]. Since miRNAs usually regulate several target genes, miRNA profiling could be a better classifier than gene expression profiling. MiR-761 is located on chromosome $1 \mathrm{p} 2$. It was found to inhibit mitochondrial fission and cardiomyocyte apoptosis by targeting mitochondrial fission factor (MFF) [14]. In addition, miR-761 was predicted to regulate the plasticity and development of hippocampal neurons [15]. However, the role of miR-761 in cancer is still unknown.

In this study, we first investigated the function of miR-761 in NSCLC tumorigenesis, and found that miR-761 was significantly upregulated in NSCLC serum and tissues. We next performed cell proliferation, wound healing, and transwell assays to examine the role of miR-761 in regulation of cell proliferation and metastatic activity of NSCLC cell lines. Mechanistically, miR-761 expression is negatively correlated with expression of ING4 (inhibitor of growth family, member 4) and TIMP2 (tissue inhibitor of metalloproteinase 2). Luciferase reporter assays and functional analyses further confirmed the role of miR-761 in regulation of NSCLC cell metastasis, which is mediated by ING4 and TIMP2.

\section{Materials and Methods}

\section{Patient samples}

A total of 33 non-small cell lung cancer (NSCLC) patients who underwent tumor resection in Department of Chest Surgery, Harbin Medical University Cancer Hospital in 2013 were recruited for this study. Among these patients, 19 participants were diagnosed adenocarcinoma and 14 patients are squamous cell carcinoma. Written informed consent was obtained from each patient. Tissue specimens were processed according to the protocols of the Ethics Committee of Harbin Medical University. Fresh tumor samples and paired adjacent normal lung tissues were immediately snap-frozen and stored at $-80^{\circ} \mathrm{C}$ for future use.

Cell cultures

A549, H23, H157, H460 and H522 human lung cancer cell lines and a normal diploid human lung fibroblast cell line, MRC5, were all purchased from the American Type Culture Collection (ATCC, Manassas, VA, USA). These cells were cultured in Dulbecco's modified Eagle medium (DMEM) or RPMI 1640 medium supplemented with 10\% fetal bovine serum (FBS) (Gibco, Rockville, MD, USA), penicillin (100 IU/ml) and Streptomycin $(100 \mu \mathrm{g} / \mathrm{ml})$ (Gibco). Cells were maintained at $37^{\circ} \mathrm{C}$ in a $5 \% \mathrm{CO}_{2}$ humidified atmosphere.

MiRNA, siRNA, plasmids, and transfection

MiR-761, miR-NC (miR-negative control), antimiR-761, antimiR-NC, ING4 siRNA, TIMP2 siRNA, and scrambled siRNA were all purchased from Santa Cruz Biotechnology (Dallas, TX, USA). These molecules were transfected into cells using Lipofectamine ${ }^{\circledR}$ RNAiMAX Transfection Reagent (Life Technologies, Gaithersburg, MD, USA) according to the manufacturer's instructions. The 3'UTR of ING4 and TIMP2 were 


\section{Cellular Physiology Cell Physiol Biochem 2015;37:55-66 \\ \begin{tabular}{l|l|l}
\hline DOI: 10.1159/000430333 & (c) 2015 S. Karger AG, Basel
\end{tabular} \\ \begin{tabular}{l|l} 
and Biochemistry Published online: August 12, 2015 & www.karger.com/cpb
\end{tabular} \\ Yan et.al.: MiR-761 Promotes NSCLC by Targeting ING4 and TIMP2}

amplified from MRC5 cells by PCR and constructed in a pMIR luciferase reporter (Promega, Madison, WI, USA). The ING4 and TIMP2 gene without 3'UTR region was PCR-amplified from MRC5 cells and inserted to pcDNA3.1 vector (Promega). Lipofectamine $₫ 2000$ was used for plasmid transfection (Life Technologies). Luciferase activity was measured $48 \mathrm{~h}$ posttransfection, using a dual luciferase reporter system (Promega) according to the manufacturer's protocol and normalized to the corresponding Renilla luciferase activity.

\section{Quantitative real-time PCR ( $q P C R$ )}

Total RNA was isolated using Trizol reagent (Life Technologies, Carlsbad, CA, USA) according to the manufacturer's recommendations. The expression levels of miR-761 were quantified using the miRNAspecific TaqMan MiRNA Assay Kit (Applied Biosystems, Foster City, CA, USA), and normalized with U6 small nuclear RNA. Total RNA (1 $\mu \mathrm{g})$ was subjected to reverse transcription with the SuperScript III RT PCR kit (Life Technologies). cDNA was applied to real-time PCR amplification with SYBR Green for ING4 and TIMP2 mRNA detection. $\beta$-actin was used as an internal control. The sequences for qRT-PCR primers were as follows:

ING4 sense primer (5'-CAAGGAATTTGGTGACGACAAG-3') and

antisense primer (5'-TCCAGCCGCCGAATGT-3');

TIMP2 sense primer (5'-GTCATCTTGATCTCATAACGCTGG-3') and

antisense primer (5'-AGCCCATCTGTACCTGTGGTTCA-3');

$\beta$-actin sense primer ( $5^{\prime}$-CAGGGAGTGATGGTGGGCA-3') and

antisense primer (5'-CAAACATCATCTGGTCATCTTCTC-3').

\section{MTT assay}

MTT assay was performed as previously described [16], briefly, cells $\left(2.5 \times 10^{3}\right)$ were seeded into 96well plates in growing medium and allowed to adhere overnight. MTT solution ( $20 \mu \mathrm{L}$; Promega) was added into medium at each time point and incubated for an additional $3 \mathrm{~h}$ at $37^{\circ} \mathrm{C}$ in the dark. OD values were measured at $450 \mathrm{~nm}$.

\section{BrdU assay}

Transfected cells were grown on 4-well chamber slides for 2 days. Medium containing BrdU $(10 \mu \mathrm{mol} / \mathrm{L})$ (Sigma, St. Louis, MO, USA) was added to each well and incubated at $37^{\circ} \mathrm{C}$ for $1 \mathrm{~h}$. Cells were washed and fixed using $4 \%$ paraformaldehyde. Cells were then incubated with anti-BrdU antibody overnight at $4{ }^{\circ} \mathrm{C}$ after blocking. Fluorescein isothiocyanate (FITC)-conjugated goat anti-mouse IgG (Dako, Carpintera, CA, USA) was used to detect BrdU incorporation. Cells were visualized under an Olympus fluorescence microscope (Olympus, Tokyo, Japan).

\section{Wound healing assay}

Treated cells $\left(1 \times 10^{6}\right)$ were initially seeded uniformly onto six-well plates and cultured overnight. An artificial homogenous "wound" was carefully created at $0 \mathrm{~h}$, using a sterile plastic 200 pipette tip to scratch the confluent cell monolayer. After wounding, the debris was removed by washing the cells with medium. Cells that migrated into the wounded area or cells were photographed and measured at 0 and $36 \mathrm{~h}$.

\section{Transwell assay}

Invasion of NSCLC cells was determined using Matrigel invasion chambers with 8 - $\mu$ m porous membranes (BD Biosciences, San Jose, CA, USA). Transfected cells $\left(2 \times 10^{5}\right)$ were plated into the upper chamber in $100 \mu \mathrm{L}$ of basal medium with $0.1 \%$ FBS. The lower chamber was filled with $400 \mu \mathrm{L}$ basal medium with $10 \%$ FBS in 24-well plates. After $24 \mathrm{~h}$, uninvaded cells in the inserts were removed with cotton swabs. The invaded cells on the bottom of the membrane were fixed and stained with $0.1 \%$ crystal violet. The numbers of invaded cells were counted.

\section{Western blot}

Cells were washed with cold PBS and lysed with RIPA buffer with protease inhibitor. Cell lysates were centrifuged at $12,000 \mathrm{rpm}$ for $15 \mathrm{~min}$ at $4^{\circ} \mathrm{C}$, and supernatants were collected. Protein concentrations were measured using the BCA kit (Pierce, Rockford, IL, USA), and equal amounts of total protein were separated by $10 \%$ sodium dodecyl sulfate polyacrylamide gel electrophoresis and transferred to polyvinylidene 
difluoride membranes (PVDF, Bio-Rad, Hercules, CA, USA), which were blocked with 5\% skimmed milk in TBS containing $0.1 \%$ Tween-20 (TBST), followed by incubation with primary antibodies overnight at $4^{\circ} \mathrm{C}$. After rinsing, membranes were incubated with secondary antibodies for $2 \mathrm{~h}$ at room temperature. Antibodies against ING4, TIMP2, and $\beta$-actin were all purchased from Santa Cruz Biotechnology.

\section{Statistical analysis}

Each experiment was performed at least three times. Statistical analysis was performed with SPSS 16.0 for Windows (SPSS, Chicago, IL, USA). The data were plotted as mean \pm SD. One-way Anova was used for group comparisons. Pearson's chi-square test was used for clinical characteristic comparisons. A $P$ value of $<0.05$ was considered statistically significant. All images were captured and measured, using Image J software.

\section{Results}

MiR-761 is overexpressed in NSCLC patients' serum, NSCLC tissues and cell lines

To investigate the role of miR-761 in human NSCLC, we first searched the public database for miR-761 expression in NSCLC patient samples and healthy controls. Analysis using database NCBI/GEO/GSE40738 [17] showed that miR-761 was significantly overexpressed in NSCLC patient serum samples (Fig. 1A, p < 0.01). This result was further confirmed by examining 33 NSCLC patient serum samples and serum from 33 normal participants from our hospital (Fig. 1B). Next, qPCR was performed to analyze miR-761 expression in NSCLC tissues, then compared them with those in adjacent normal lung tissue. Results showed that miR-761 was significantly overexpressed in NSCLC tissues (Fig. 1C). Furthermore,

A

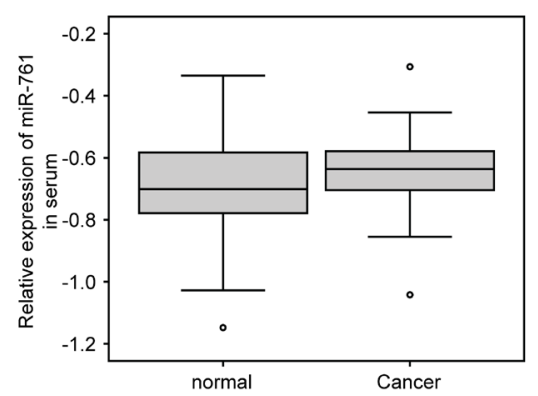

C

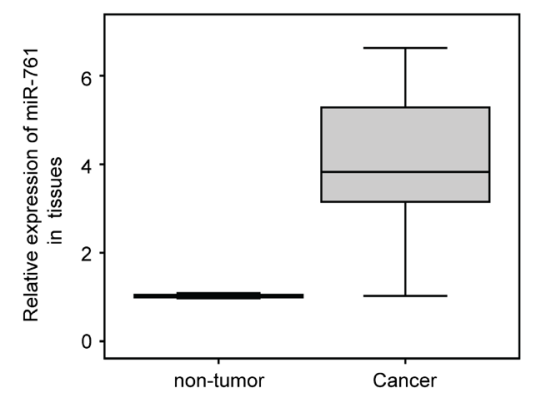

B

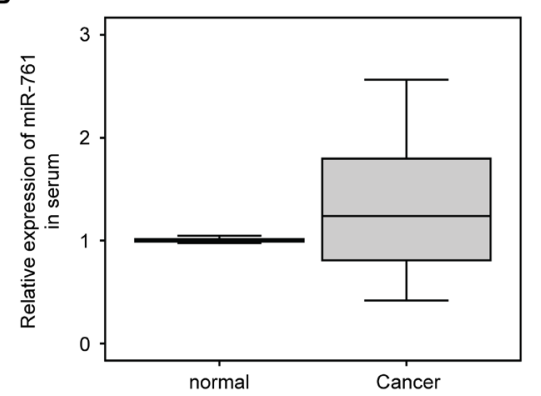

D

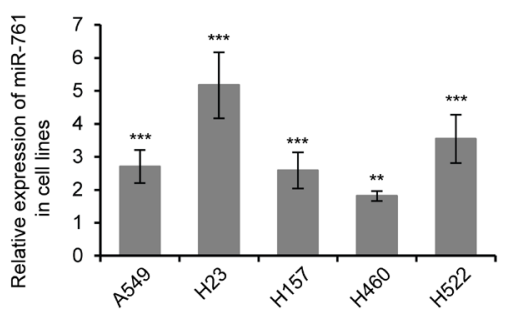

Fig. 1. Upregulation of miR-761 in NSCLC patients' serum, NSCLC tissues, and NSCLC cell lines. (A) qPCR analysis of miR-761 in serum of 86 NSCLC patients compared to 77 normal participants (GSE40738, $p=0.001$ ). (B) qPCR analysis of miR-761 levels in the serum of 33 NSCLC patients compared to that in 33 normal participants $(p<0.001)$. (C) qPCR analysis of miR-761 expression in 33 NSCLC samples and paired adjacent non-tumor tissues ( $p<0.001)$. (D) Expression levels of miR-761 in five NSCLC cell lines (A549, H23, H157, H460 and H522) when compared to a normal diploid human lung fibroblast cell line, MRC5 $\left({ }^{* *} p<0.01\right.$, $* * * p<0.001)$. 


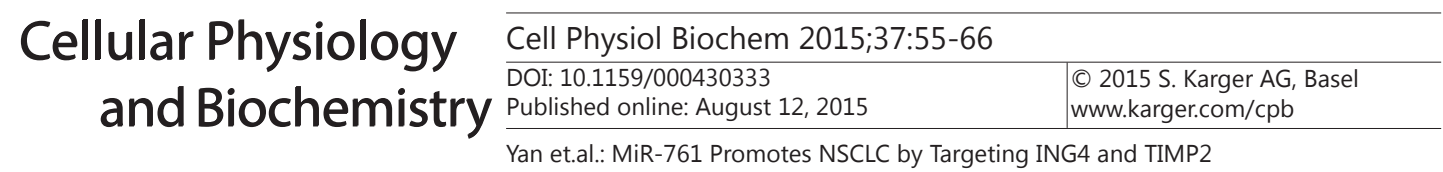

Table 1. Clinical characteristics of 33 NSCLC patients

\begin{tabular}{|c|c|c|c|c|}
\hline Variables & No. of patients & $\begin{array}{c}\text { miR-761 } \\
\text { low expression }\end{array}$ & $\begin{array}{c}\text { miR-761 } \\
\text { high expression }\end{array}$ & $p$ value \\
\hline Gender & & & & 0.017 \\
\hline Male & 22 & 8 & 14 & \\
\hline Female & 11 & 9 & 2 & \\
\hline Smoking & & & & 0.051 \\
\hline Never & 7 & 6 & 1 & \\
\hline Ever & 26 & 11 & 15 & \\
\hline Age & & & & 0.563 \\
\hline$<60$ & 15 & 8 & 7 & \\
\hline$\geq 60$ & 18 & 9 & 9 & \\
\hline Differentiation & & & & 0.001 \\
\hline Well/moderate & 12 & 11 & 1 & \\
\hline Poor & 21 & 6 & 15 & \\
\hline Histology type & & & & 0.420 \\
\hline Adenocarcinoma & 19 & 9 & 10 & \\
\hline Squamous cell carcinoma & 14 & 8 & 6 & \\
\hline Lymph node status & & & & 0.001 \\
\hline Negative & 9 & 9 & 0 & \\
\hline Positive & 24 & 8 & 16 & \\
\hline
\end{tabular}

A

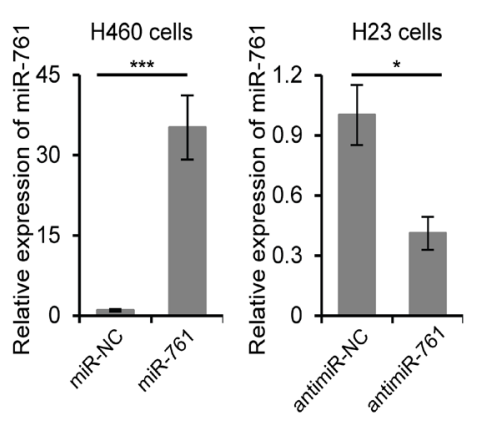

B

H460 cells
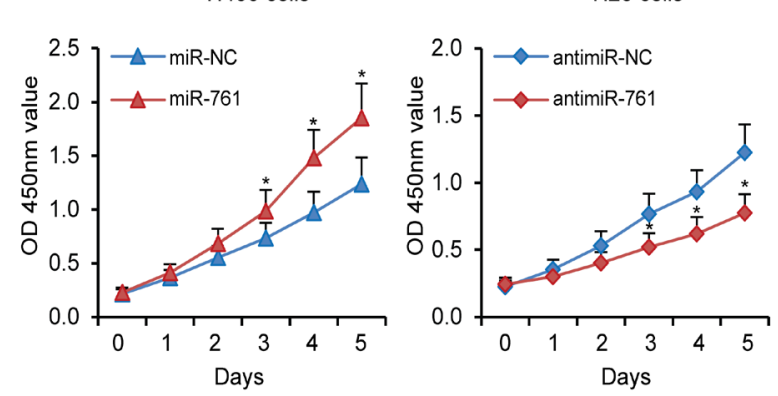

C
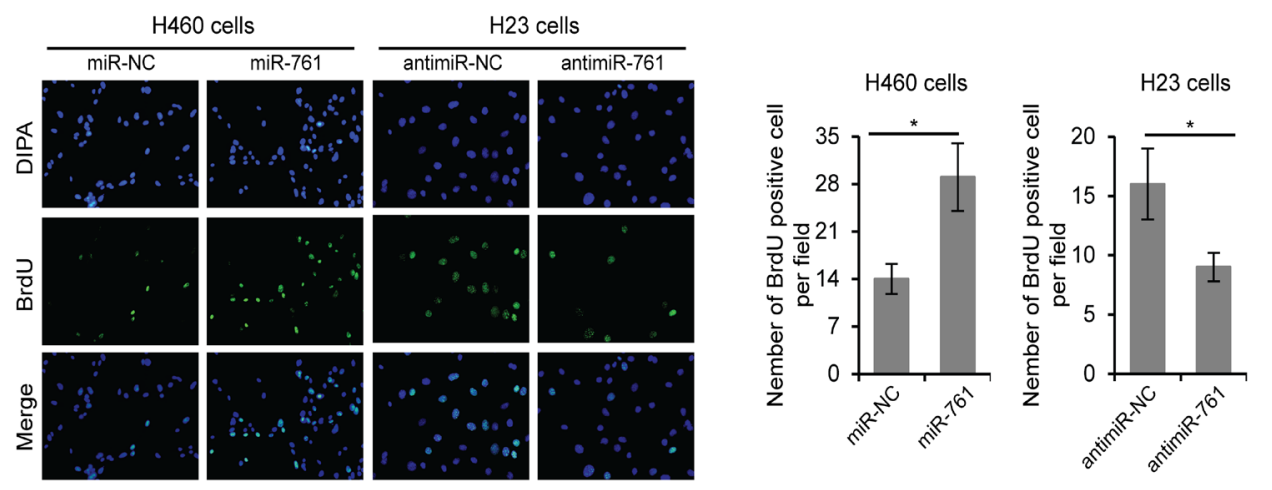

Fig. 2. MiR-761 promotes NSCLC cell proliferation. (A) Relative expression of miR-761 analyzed by qPCR. H460 cells were transfected with miR-NC or miR-761 and H23 cells transfected with antimiR-NC or antimiR-761 for $48 \mathrm{~h}$ before the analysis. (B) MTT assays at indicated time points after transfection. (C) BrdU cell proliferation assay. ${ }^{*} p<0.05,{ }^{* * *} p<0.001$ when compared to miR-NC- or antimiR-NC-transfected cells.

NSCLC tissues with poor differentiation exhibited significantly higher expression of miR761 as compared to well/moderate differentiation of NSCLC samples (Table 1), suggesting promotive role of miR-761 in NSCLC development. Lastly, we examined miR-761 expression in NSCLC cell lines. Consistently, miR-761 was upregulated in all five NSCLC cell lines tested 
A
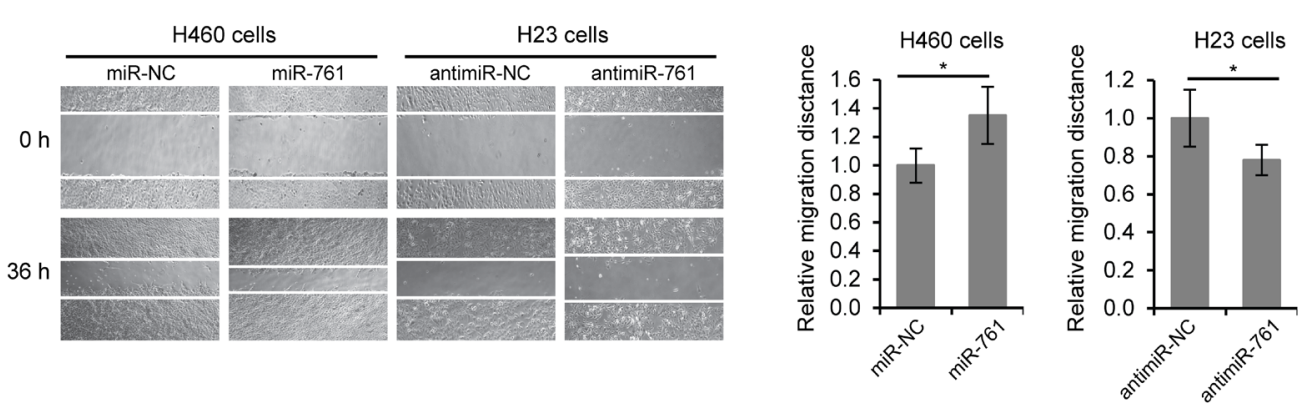

B
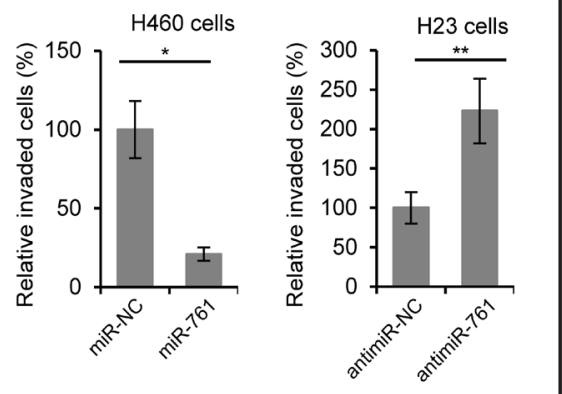

Fig. 3. MiR-761 stimulates cell migration and invasion in NSCLC cells. (A-B) Wounded healing (A) and transwell (B) assays. H460 cells were transfected with negative control miRNA (miR-NC) or miR-761, and H23 cells were transfected with negative control anti-miRNA (antimiR-NC) or antimiR-761. Assays were performed $48 \mathrm{~h}$ after transfection. Relative migration distance and invaded cells are shown as mean \pm SD. ${ }^{*} p<0.05,{ }^{* *} p<0.01$ when compared to miR-NC-transfected cells or antimiR-NC-transfected cells.

compared to normal diploid human lung fibroblast cell line, MRC5 (Fig. 1D). We selected a miR-761 high-expression cell line, H23, and low-expression cell line, H460, for further experiments.

\section{MiR-761 promotes NSCLC cell proliferation, migration and invasion}

To evaluate the role of miR-761 in NSCLC development, we transiently transfected miR761 or miR-761 inhibitor (antimiR-761) into H460 or H23 NSCLC cells, respectively (Fig. 2A). In the H460 cell line, MTT assay results revealed a significant increase in cell proliferation when miR-761 was overexpressed, while inhibition of miR-761 expression in H23 cells led to significantly reduced cell proliferation (Fig. 2B). BrdU assays further confirmed these results. More BrdU-positive cells were observed in response to ectopic expression of miR761 in H460 cells (Fig. 2C). We next examined the effects of miR-761 on NSCLC cell migration and invasion ability. The wound healing assay revealed that upregulation of miR-761 induced faster closing of scratch wounds in H460 cells, and inhibition of miR-761 resulted in slower closing of scratch wounds in H23 cells (Fig. 3A). Similarly, in transwell invasion assays, overexpression of miR-761 remarkably enhanced invasion of H460 cells. Likewise, antimiR-761 significantly inhibited the invasion of H23 cells (Fig. 3B). Moreover, we found high expression of miR-761 was correlated with lymphatic metastasis in NSCLC patient samples (Table 1). These results indicate that miR-761 regulates the proliferation, migration and invasion of NSCLC.

\section{MiR-761 directly targets ING4 and TIMP2}

To investigate the mechanisms by which miR-761 mediates increases in NSCLC cell proliferation and motility, we used TargetScan algorithm and resources from microRNA.org and found ING4 and TIMP2 are potential targets of miR-761 (Fig. 4A). We inserted WT (wild type) or MU (mutant) 3'UTRs of ING4 and TIMP2 downstream of the luciferase reporter 


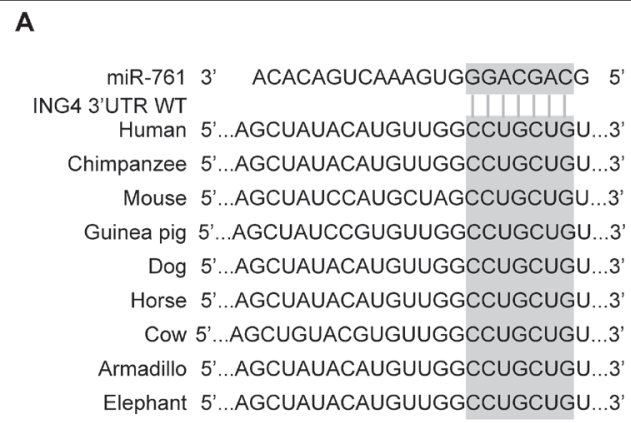

A

miR-761 3' ACACAGUCAAAGUGGGACGACG 5 ING4 3'UTR WT

Human $5^{\prime}$...AGCUAUACAUGUUGGCCUGCUGU....3' Chimpanzee 5'...AGCUAUACAUGUUGGCCUGCUGU...3'

Mouse 5 '...AGCUAUCCAUGCUAGCCUGCUGU....3' Guinea pig 5'...AGCUAUCCGUGUUGGCCUGCUGU...3'

Dog 5 '...AGCUAUACAUGUUGGCCUGCUGU...3'

Horse $5^{\prime}$...AGCUAUACAUGUUGGCCUGCUGU....3'

Cow $5^{\prime}$...AGCUGUACGUGUUGGCCUGCUGU....3'

Armadillo 5 '...AGCUAUACAUGUUGGCCUGCUGU... 3'

Elephant 5 '...AGCUAUACAUGUUGGCCUGCUGU ... 3 '

miR-761 3' ACACAGUCAAAGUGGGACGACG 5' ING4 3'UTR MU 5'...AGCUAUACAUGUUGGCCUGACGU....3'

B
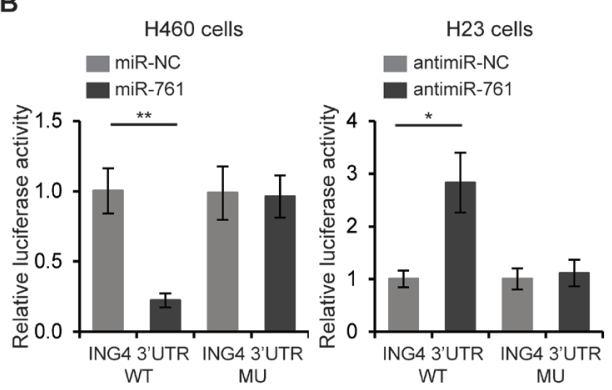

D
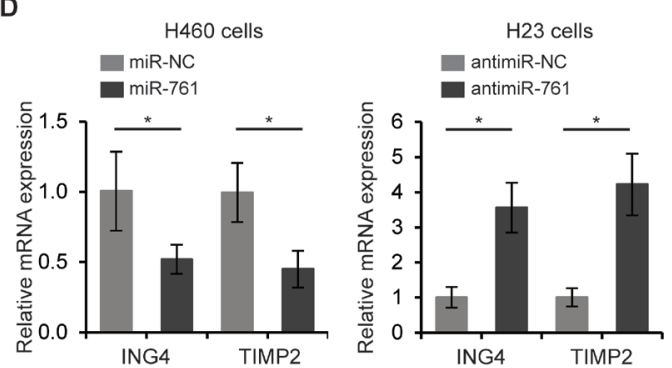

miR-761 3' ACACAGUCAAAGUGGGACGACG 5 TIMP2 3'UTR WT

Human 5'...CAUUUACUCCUGUUUCUGCUGAU...3'

Chimpanzee 5 '...CAUUUACUCCUGUUUCUGCUGAU....3'

Mouse 5 '...UAACAGUGCCUGUUUCUGCUGAU... $3^{\prime}$

Guinea pig 5 '...CAUUUGCUCCUGUUUCUGCUGAC ...3'

Dog 5'...CAUUUACUCCUGUUUCUGCUGAU.... $3^{\prime}$

Horse $5^{\prime}$ '...CAUUUACUCCUGUUUCUGCUGAU.... $3^{\prime}$

COW 5'...CAUUUACUCCUGUUUCUGCUGAU.... $3^{\prime}$

Armadillo 5'...CAUUUACUCCUGGUUCUGCUGAC....3'

Elephant 5 '...CAUUUACUCCUGUUUCUGCUGAU....3'

miR-761 3 ' ACACAGUCAAAGUGGGACGACG 5 '

TIMP2 3'UTR MU 5'...CAUUUACUCCUGUUUCUGCGACU... 3'

C

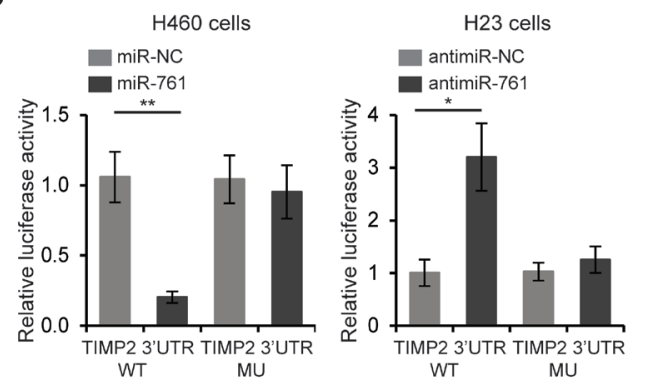

E

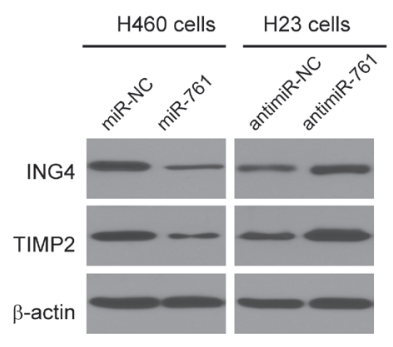

Fig. 4. MiR-761 directly targets ING4 and TIMP2. (A) Schematic representation of predicted binding sites between the miR-761 seed and the seed matches on human the 3'UTRs of ING4 and TIMP2. (B) Luciferase assays in H460 and H23 cells co-transfected with indicated miRNAs and luciferase reporter plasmids carrying wild type (WT) or mutant (MU) ING4 3'UTR. Four nucleotides (dotted lines) were mutated in the predicted binding sites of miR-761 on the ING4 3'UTR. (C) Luciferase assays of H460 and H23 cells cotransfected with indicated miRNAs and luciferase reporter plasmids carrying WT or MU TIMP2 3'UTR. Four nucleotides (dotted lines) were mutated in the predicted binding sites of miR-761 on the TIMP2 3'UTR. qPCR (D) and Western blot (E) analysis of ING4 and TIMP2 expression in H460 transfected with control or miR-761, and H23 cells transfected with antimiR-NC and antimiR-761. ${ }^{*} p<0.05,{ }^{* *} p<0.01$ when compared to miR-NC-transfected cells or antimiR-NC-transfected cells.

gene in the pMIR reporter plasmid (Fig. 4B and 4C). These constructs were co-transfected with miR-761 into H460 cells or with antimiR-761 into H23 cells for luciferase reporter assays. As shown in Fig. 4B and 4C, miR-761 reduced the luciferase activity of the plasmid carrying WT but not MU ING4 or the TIMP2 3' UTR in H460 cells. Additionally, inhibition of miR-761 increased the luciferase activity of the plasmid carrying the WT $3^{\prime}$ UTRs of ING4 and TIMP2 in H23 cells. Subsequently, ING4 and TIMP2 mRNA and protein levels were found to be significantly decreased in NSCLC cells by miR-761 and increased by antimiR-761 (Fig. 4D and 4E). Furthermore, negative correlation between miR-761 and ING4 or TIMP2 in 33 
Fig. 5. Expression of miR-761 is negatively correlated with ING4 and TIMP2 in NSCLC. Spearman correlation analysis between miR-761 and ING4 ( $\mathrm{r}=$ -0.66) (A) or between miR-761 and TIMP2 ( $\mathrm{r}$ $=-0.71$ ) (B) in 33 clinical NSCLC samples.

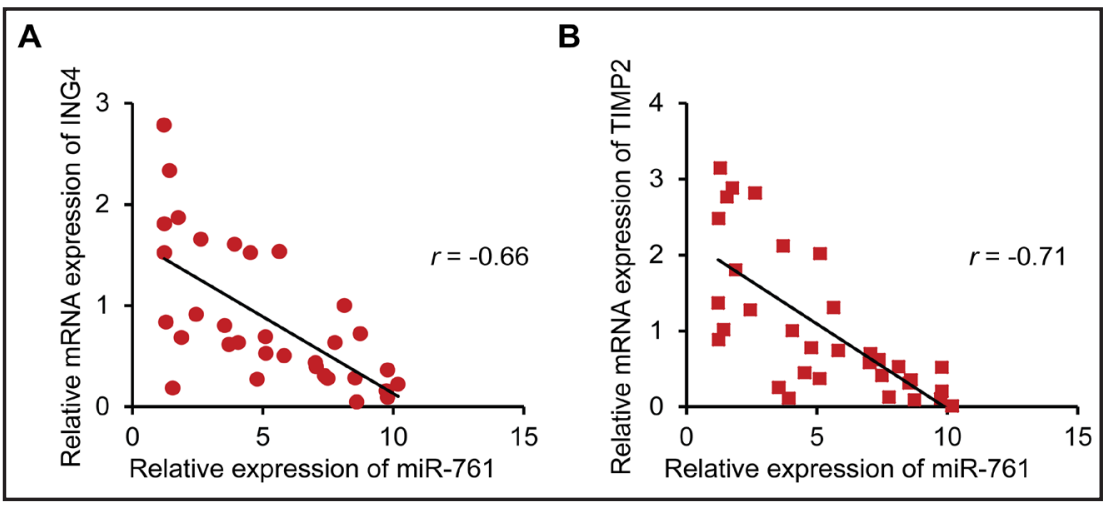

\section{A}

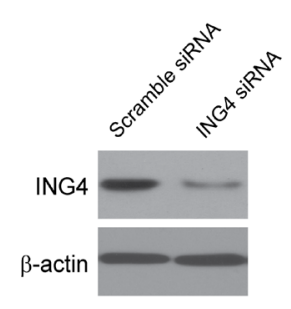

E

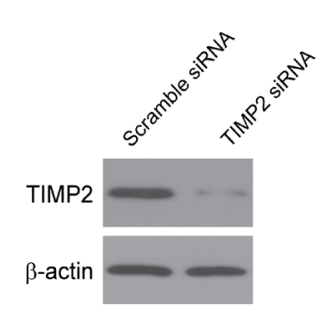

B

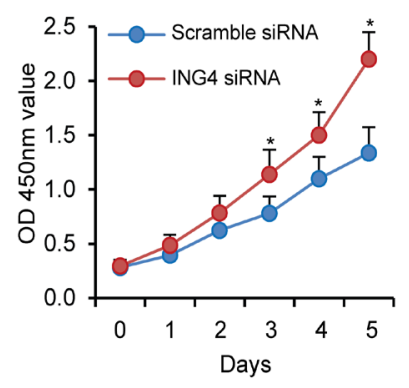

F

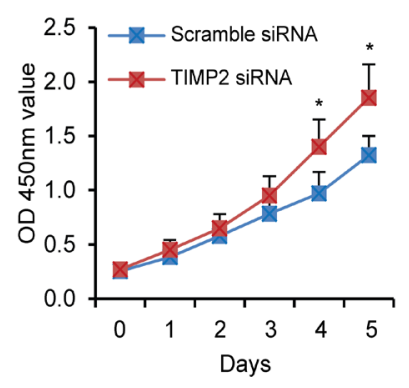

C

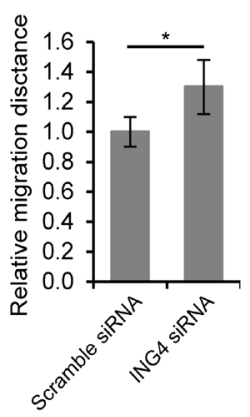

G

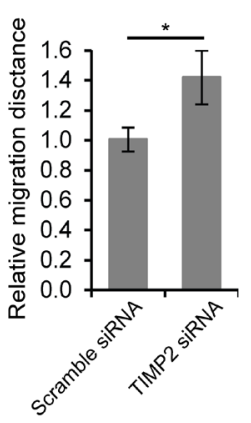

D

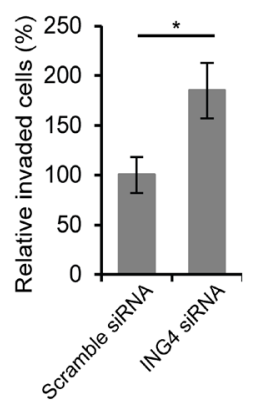

H

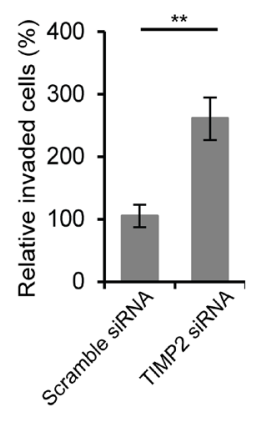

Fig. 6. ING4 and TIMP2 suppress NSCLC cell proliferation and motility. (A) Western blot of ING4 expression in H460 cells transfected with ING4 siRNA or scrambled siRNA. (B-D) MTT (B), wound healing (C), and transwell (D) assays in H460 cells transfected with ING4 siRNA or scrambled siRNA. (E) Western blot of ING4 expression in H460 cells transfected with TIMP2 siRNA or scrambled siRNA. (F-H) MTT (F), wound healing $(\mathrm{G})$, and transwell $(\mathrm{H})$ assays in H460 cells transfected with TIMP2 siRNA or scrambled siRNA. ${ }^{*} p<0.05,{ }^{* *} p<0.01$ when compared to scrambled siRNA-transfected cells.

clinical NSCLC samples confirmed that ING4 and TIMP2 are potential targets of miR-761 (Fig. 5A and 5B). Together, miR-761 downregulates ING4 and TIMP2 expression through direct binding to their $3^{\prime}$-UTR, and the expressions of miR-761 and ING4 or TIMP2 mRNA reveal inverse correlation in NSCLC tissues.

\section{MiR-761 regulates NSCLC cell proliferation and metastasis via ING4 and TIMP2}

It has been reported that ING4 and TIMP2 play negative roles in lung cancer development $[18,19]$. To further confirm their functions in NSCLC cell lines, specific siRNAs were used to knock down ING4 and TIMP2 in H460 cells. Similar to overexpression of miR-761 in H460 cells, depletion of ING4 or TIMP2 significantly stimulated cell proliferation, migration, and invasion ability (Fig. 6A-H). To investigate whether the role of miR-761 in tumor promotion 
A

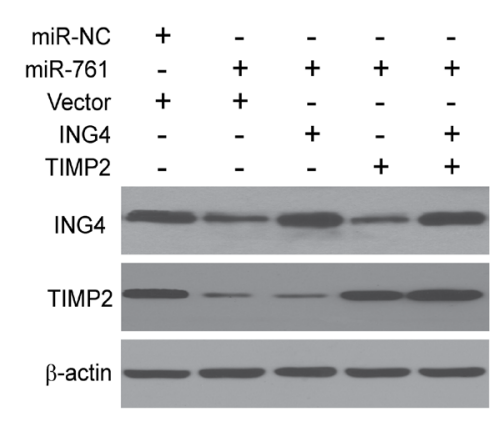

C

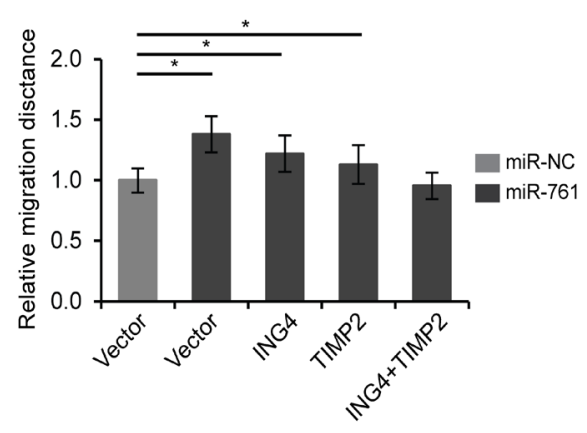

B

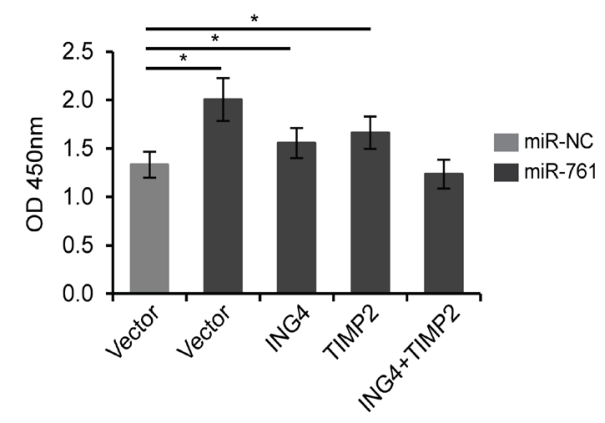

D

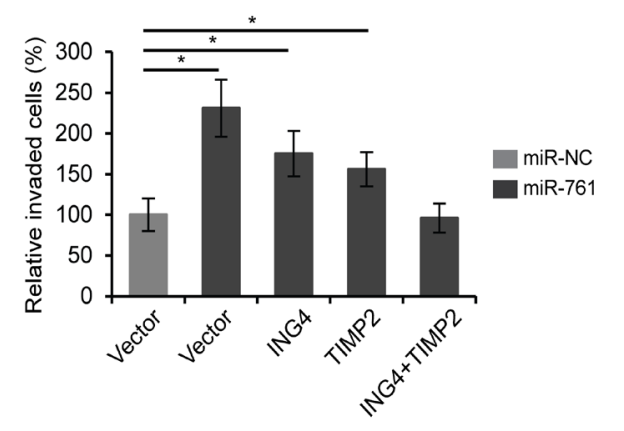

Fig. 7. ING4 and TIMP2 mediate the role of miR-761 in regulation of NSCLC tumorigenesis. (A) Western blot analysis of ING4 and TIMP2 expression in H460 cells co-transfected with indicated miRNA and plasmids. $\beta$-actin was used as loading control. MTT (B), migration (C), and invasion (D) assays in H460 cells cotransfected with indicated miRNA and plasmids. ${ }^{*} p<0.05$ when compared to miR-NC- and pcDNA3.1 vectortransfected cells.

is mediated by ING4 and TIMP2, we co-overexpressed ING4 and/or TIMP2 with miR-761 in H460 cells (Fig. 7A). MTT, wound healing, and transwell assays were subsequently performed. As showed in Fig. 7B-D, overexpression of ING4 or TIMP2 abrogated the enhancement effects of miR-761 in NSCLC cell proliferation, migration and invasion, at least in part. Moreover, when both ING4 and TIMP2 were overexpressed at the same time, no significant differences were observed between control cells and the cells that ectopically expressed miR-761 (Fig. 7B-D). These results suggested that miR-761 plays a role in proliferation and metastasis of NSCLC by targeting ING4 and TIMP2.

\section{Discussion}

Despite decades of progress in treatment of lung cancer, the five-year survival rate of lung cancer remains approximately $15.7 \%$ [2, 20]. According to the National Cancer Institute's Surveillance, Epidemiology, and End Results (SEER) database, the survival rates of NSCLC patients vary dramatically according to the stages at which NSCLC is diagnosed [21]. However, NSCLC is often insidious, causing no symptoms until the disease is well advanced $[22,23]$. About $70 \%$ of patients with lung cancer already have metastatic disease at the time of diagnosis [5]. Hence, there is a high demand for biomarkers that predict NSCLC progression and can be targeted for treating NSCLC.

In the present study, we found that miR-761 is significantly upregulated in NSCLC tissues as compared to paired adjacent normal lung tissue. Compared to normal control, NSCLC patients' serum with exhibited higher expression of miR-761. High expression of miR- 
761 is associated with poor differentiation of NSCLC cells. Additionally, miR-761 expression is negatively correlated with ING4 and TIMP2 [24]. We propose that miR-761 may serve as a biomarker of progression and metastasis in patients with NSCLC. However, miR-761 may have different functions in other types of cancer.

To determine the potential role of miR-761 overexpression in NSCLC, we performed MTT assays and BrdU staining, which showed a higher proliferation rate in $\mathrm{H} 460$ cells with ectopic expression of miR-761. Further, the results of the wound healing and transwell assays demonstrated that overexpression of miR-761 enhanced metastatic activity of H460 cells. Conversely, treatment with the miR-761 inhibitor resulted in a decrease in cell proliferation and metastatic activity of $\mathrm{H} 23$ cells. These results suggest that miR-761 overexpression could contribute to progression and metastasis of NSCLC. However, in vivo studies are needed to determine the stage at which miR-761 expression starts to rise, as well as the role of miR761 during development of NSCLC. We also need a better understanding of the effects of miR-761 on NSCLC cells, such as anti-apoptosis. Jonathan E. Cohen has predicted miR-761 regulates networks of gene important in plasticity, development and tumorigenesis. We first study the biofunctional role of miR-761 in NSCLC.

We identified ING4 and TIMP2 as the direct targets of miR-761. After using the predictive TargetScan algorithm and resources from microRNA.org [25], luciferase reporter assays were performed, and the results revealed that miR-761 directly bound to the 3'UTR in the mRNA of ING4 and TIMP2. Furthermore, functional studies were performed to confirm whether the role of miR-761 is mediated by ING4 and TIMP2. Overexpression of ING4 and TIMP2 consistently reduced cell metastatic activity to the basal level, which was enhanced by ectopic expression of miR-761 in H460 cells. ING4 has been well-established as a tumor suppressor in gliomas, breast tumors, stomach adenocarcinoma, and lung cancers [2629]. Wang et al. reported that downregulation of ING4 was associated with initiation and progression of lung cancer [28]. In our study, we confirmed that siRNA-mediated depletion of ING4 significantly increased cell proliferation and metastatic activity in H460 cells. TIMP2 was found to inhibit metastasis by enhancing E-cadherin/beta-catenin complex expression in A549 lung cancer cells [19]. Similar to ING4, depletion of TIMP2 promoted H460 cell proliferation, metastasis and invasion. Additionally, adenovirus-mediated overexpression of ING4 was found to enhance the radiosensitivity of nonsmall cell lung cancer [30], and to overcome docetaxel resistance in lung adenocarcinoma [31]. Taken together, it seems that inhibition of miR-761 suppresses progression and metastasis of NSCLC, and facilitates docetaxel-based chemotherapies and radiotherapies of NSCLC.

In summary, we found that miR-761 expression was upregulated in NSCLC serum and tissues. Mechanistically, miR-761 promoted cell proliferation, metastasis and invasion by targeting ING4 and TIMP2 in NSCLC cell lines. Our findings indicate that miR-761 may be a biomarker and a target for gene therapies for NSCLC.

\section{Acknowledgements}

This work was supported by the National Natural and by Natural Science Foundation of Heilongjiang Province, China (grant number: LC2012C08 to M.Q.) and the Science Foundation of China (grant number 30772540, 81172214 to L.C.).

\section{Disclosure Statement}

None. 


\section{Cellular Physiology Cell Physiol Biochem 2015;37:55-66 \begin{tabular}{l|l}
\hline DOI: 10.1159/000430333 & (c) 2015 S. Karger AG, Base \\
wwy Biochemistry
\end{tabular} \\ Yan et.al.: MiR-761 Promotes NSCLC by Targeting ING4 and TIMP2}

\section{References}

1 Ettinger DS, Akerley W, Borghaei H, Chang AC, Cheney RT, Chirieac LR, D'Amico TA, Demmy TL, Ganti AK, Govindan R, Grannis FW, Jr., Horn L, Jahan TM, Jahanzeb M, Kessinger A, Komaki R, Kong FM, Kris MG, Krug LM, Lennes IT, Loo BW, Jr., Martins R, O'Malley J, Osarogiagbon RU, Otterson GA, Patel JD, Pinder-Schenck MC, Pisters KM, Reckamp K, Riely GJ, Rohren E, Swanson SJ, Wood DE, Yang SC, Hughes M, Gregory KM, NCCN: Non-small cell lung cancer. J Natl Compr Canc Netw 2012;10:1236-1271.

2 Molina JR, Yang P, Cassivi SD, Schild SE, Adjei AA: Non-small cell lung cancer: Epidemiology, risk factors, treatment, and survivorship. Mayo Clin Proc 2008;83:584-594.

3 Peters S, Adjei AA, Gridelli C, Reck M, Kerr K, Felip E, Group EGW: Metastatic non-small-cell lung cancer (nsclc): Esmo clinical practice guidelines for diagnosis, treatment and follow-up. Ann Oncol 2012;23:vii5664.

4 Rusch VW, Asamura H, Watanabe H, Giroux DJ, Rami-Porta R, Goldstraw P, Members of ISC: The iaslc lung cancer staging project: A proposal for a new international lymph node map in the forthcoming seventh edition of the tnm classification for lung cancer. J Thorac Oncol 2009;4:568-577.

5 Beckles MA, Spiro SG, Colice GL, Rudd RM: Initial evaluation of the patient with lung cancer: Symptoms, signs, laboratory tests, and paraneoplastic syndromes. Chest 2003;123:97S-104S.

6 Cornfield J, Haenszel W, Hammond EC, Lilienfeld AM, Shimkin MB, Wynder EL: Smoking and lung cancer: Recent evidence and a discussion of some questions. 1959. Int J Epidemiol 2009;38:1175-1191.

7 Toh CK, Gao F, Lim WT, Leong SS, Fong KW, Yap SP, Hsu AA, Eng P, Koong HN, Thirugnanam A, Tan EH: Never-smokers with lung cancer: Epidemiologic evidence of a distinct disease entity. J Clin Oncol 2006;24:2245-2251.

8 Stefani G, Slack FJ: Small non-coding rnas in animal development. Nat Rev Mol Cell Biol 2008;9:219-230.

9 Hu X, Zhang F, Liu XR, Wu YT, Ni YM: Efficacy and potential microrna mechanism for computed tomography-guided percutaneous radiofrequency ablation of primary lung cancer and lung metastasis from liver cancer. Cell Physiol Biochem 2014;33:1261-1271.

10 Zhang H, Zhao M, Lv Z, Zhang X, Qin X, Wang H, Wang S, Su J, Lv X, Liu H, Du W, Zhou W, Chen X, Fei K: Mir138 inhibits tumor growth through repression of ezh2 in non-small cell lung cancer. Cell Physiol Biochem 2013;31:56-65.

11 Wang J, Yang B, Han L, Li X, Tao H, Zhang S, Hu Y: Demethylation of mir-9-3 and mir-193a genes suppresses proliferation and promotes apoptosis in non-small cell lung cancer cell lines. Cell Physiol Biochem 2013;32:1707-1719.

12 Guz M, Rivero-Muller A, Okon E, Stenzel-Bembenek A, Polberg K, Slomka M, Stepulak A: Micrornas-role in lung cancer. Dis Markers 2014;2014:218169.

13 Yanaihara N, Caplen N, Bowman E, Seike M, Kumamoto K, Yi M, Stephens RM, Okamoto A, Yokota J, Tanaka T, Calin GA, Liu CG, Croce CM, Harris CC: Unique microrna molecular profiles in lung cancer diagnosis and prognosis. Cancer Cell 2006;9:189-198.

14 Long B, Wang K, Li N, Murtaza I, Xiao JY, Fan YY, Liu CY, Li WH, Cheng Z, Li P: Mir-761 regulates the mitochondrial network by targeting mitochondrial fission factor. Free Radic Biol Med 2013;65:371-379.

15 Cohen JE, Lee PR, Fields RD: Systematic identification of 3'-utr regulatory elements in activity-dependent mrna stability in hippocampal neurons. Philos Trans R Soc Lond B Biol Sci 2014;369

16 Xia Y, Chen Q Zhong Z, Xu C, Wu C, Liu B, Chen Y: Down-regulation of mir-30c promotes the invasion of non-small cell lung cancer by targeting mta1. Cell Physiol Biochem 2013;32:476-485.

17 Raponi M, Dossey L, Jatkoe T, Wu X, Chen G, Fan H, Beer DG: Microrna classifiers for predicting prognosis of squamous cell lung cancer. Cancer Res 2009;69:5776-5783.

18 Xie Y, Zhang H, Sheng W, Xiang J, Ye Z, Yang J: Adenovirus-mediated ing4 expression suppresses lung carcinoma cell growth via induction of cell cycle alteration and apoptosis and inhibition of tumor invasion and angiogenesis. Cancer Lett 2008;271:105-116.

19 Bourboulia D, Han H, Jensen-Taubman S, Gavil N, Isaac B, Wei B, Neckers L, Stetler-Stevenson WG: Timp-2 modulates cancer cell transcriptional profile and enhances e-cadherin/beta-catenin complex expression in a549 lung cancer cells. Oncotarget 2013;4:166-176.

20 Siegel R, Ma J, Zou Z, Jemal A: Cancer statistics, 2014. CA Cancer J Clin 2014;64:9-29.

21 Lag R, Eisner M, Kosary C, Hankey BF, Miller BA, Clegg L, Edwards BK (eds): Cancer statistics review, 19752002. Bethesda, MD: National Cancer Institute 2005;2005. 


\section{Cellular Physiology and Biochemistry}

22 Chiramannil A: Clinical snapshot: lung cancer. Am J Nurs 1998;98:46-47.

23 Roberts PF, Straznicka M, Lara PN, Lau DH, Follette DM, Gandara DR, Benfield JR: Resection of multifocal non-small cell lung cancer when the bronchioloalveolar subtype is involved. J Thorac Cardiovasc Surg 2003;126:1597-1601.

24 Iniesta P, Moran A, De Juan C, Gomez A, Hernando F, Garcia-Aranda C, Frias C, Diaz-Lopez A, RodriguezJimenez FJ, Balibrea JL, Benito M: Biological and clinical significance of mmp-2, mmp-9, timp-1 and timp-2 in non-small cell lung cancer. Oncol Rep 2007;17:217-223.

25 Lewis BP, Burge CB, Bartel DP: Conserved seed pairing, often flanked by adenosines, indicates that thousands of human genes are microrna targets. Cell 2005;120:15-20.

26 Kim S, Chin K, Gray JW, Bishop JM: A screen for genes that suppress loss of contact inhibition: Identification of ing4 as a candidate tumor suppressor gene in human cancer. Proc Natl Acad Sci U S A 2004;101:1625116256.

27 Garkavtsev I, Kozin SV, Chernova O, Xu L, Winkler F, Brown E, Barnett GH, Jain RK: The candidate tumour suppressor protein ing4 regulates brain tumour growth and angiogenesis. Nature 2004;428:328-332.

28 Wang QS, Li M, Zhang LY, Jin Y, Tong DD, Yu Y, Bai J, Huang Q, Liu FL, Liu A, Lee KY, Fu SB: Down-regulation of ing4 is associated with initiation and progression of lung cancer. Histopathology 2010;57:271-281.

29 Doyon Y, Cayrou C, Ullah M, Landry AJ, Cote V, Selleck W, Lane WS, Tan S, Yang XJ, Cote J: Ing tumor suppressor proteins are critical regulators of chromatin acetylation required for genome expression and perpetuation. Mol Cell 2006;21:51-64.

30 Ling C, Xie Y, Zhao D, Zhu Y, Xiang J, Yang J: Enhanced radiosensitivity of non-small-cell lung cancer (nsclc) by adenovirus-mediated ing4 gene therapy. Cancer Gene Ther 2012;19:697-706.

31 Wang R, Huang J, Feng B, De W, Chen L: Identification of ing4 (inhibitor of growth 4) as a modulator of docetaxel sensitivity in human lung adenocarcinoma. Mol Med 2012;18:874-886. 